\title{
The effect of age, anatomical site and bone structure on osteogenesis in New Zealand White rabbit
}

\author{
Francesca Ravanetti, ${ }^{1}$ Edoardo Scarpa, ${ }^{1}$ Vittorio Farina, ${ }^{2}$ Marco Zedda, ${ }^{2}$ Carlo Galli, ${ }^{3}$ \\ Paolo Borghetti, ${ }^{1}$ Antonio Cacchioli ${ }^{1}$ \\ ${ }^{1}$ Department of Veterinary Science, University of Parma; ${ }^{2}$ Department of Veterinary Medicine, \\ University of Sassari; ${ }^{3}$ Department of Biomedical, Biotechnological and Translational Sciences, \\ University of Parma, Italy
}

\begin{abstract}
Among animal models, rabbits are widely used in medical research, as they fill the gap between smaller models, commonly employed in basic science, and larger ones, which are better suited for preclinical trials. Given their rapid growth, rabbits provide a valuable system for the evaluation of bone implants for tissue regeneration. By means of a histomorphometric analysis, here we quantified the mineral apposition rates (MARs) in osteonic, periosteum and endosteum osteogenic fronts, of skeletal elements within femur, tibia, radius, ulna, frontal
\end{abstract}

Correspondence: Vittorio Farina, Department of Veterinary Medicine, University of Sassari, via Vienna 2, 07100 Sassari, Italy

Tel: +39079 229460 - Fax: +39 079229432 .

E-mail: vfarina@uniss.it

Key words: Mineral apposition rate; Osteogenesis; New Zealand White rabbits; Bone fluorochrome.

Conflict of interest: the authors declare no potential conflict of interest.

Funding: this work was partially supported by CARIPARMA Foundation (Grant number: 2010.0438).

Contributions: all authors participated sufficiently in this study to take public responsibility for the content. FR was responsible for planning and conducting the experiments and histology. ES assisted FR in the maintaining the experimental animals and reading the samples. VF helped to improve the significance of the study and participated in manuscript composition. MZ analyzed samples and helped interpret the data in two different labs. CG performed statistical analysis and contributed to paper drafting. $\mathrm{PB}$ participated in manuscript composition and reviewing. AC designed the study, supervised the research team, conducted the statistical analysis of the data and reviewed the manuscript.

Received for publication: 19 December 2014.

Accepted for publication: 24 February 2015.

(C) Copyright F. Ravanetti et al., 2015

Licensee PAGEPress, Italy

Journal of Biological Research 2015; 88:4916

This article is distributed under the terms of the Creative Commons Attribution Noncommercial License (by-nc 3.0) which permits any noncommercial use, distribution, and reproduction in any medium, provided the original author(s) and source are credited. and parietal bones in New Zealand White rabbits aged 6, 7 and 8 months. Our hypothesis is that the MAR varies according to the skeletal maturity of the animal, and also within the skeletal elements and the osteogenic fronts considered. In the present study we show that the MAR in both femur and tibia is significantly higher than in ulna and radius. We also demonstrate that the MAR in parietal bones is significantly higher compared to the MAR of both frontal and forelimb bones. Contrary to what was expected, the MARs of all the skeletal elements considered were not decreased following full skeletal maturity. Finally, the MAR of the osteonic-osteogenic front is the lowest in all of the skeletal elements considered. In conclusion, these results provide new important insights for the evaluation of bone implants, casting a light on the role of both age and osteogenic fronts on the bone MAR, and providing valuable information on the physiological bone turnover in New Zealand White rabbits.

\section{Introduction}

Given the intermediate size, the long lifespan, and moderate economic cost related to its maintenance, the rabbit is widely used in medical research. In particular, due to its fast skeletal growth the rabbit is used in around 35\% of musculoskeletal studies ${ }^{1}$ and represents an excellent model for the investigation of bone implants for tissue regeneration. ${ }^{2}$

The rabbit reaches its skeletal maturity at the age of 6 months,${ }^{3}$ but the closure of the bone growth plate can vary across bones and also between different epiphyses of the same bone. ${ }^{4}$ The average age of growth plate closure is 5.3 months (22.71 weeks) for the femur and 6.8 months (29.14 weeks) for the tibia. ${ }^{5}$ According to radiographic and histological evidence, New Zealand White rabbits achieve the closure of the growth plates at 19-24 weeks in the distal femur, 22-32 weeks in the proximal tibia and $23-32$ weeks in the proximal fibula. ${ }^{6}$ Overall, rabbits grow rapidly between 15 and 43 days after birth, thus the greater increase in their bone size occurs in this early period. On the contrary, at the onset of day 50 after birth, there is an attenuation in bone length growth that reaches its minimum between day 78 and day 92. ${ }^{7}$ The femur in particular, undergoes a steep longitudinal growth reaching $97 \%$ of its adult length within 130 days, followed by a continuous decrease of the skeletal growth that ends around the age of 6 months. ${ }^{4,8}$ A similar pattern of rapid growth is observed in the craniofacial region which develops rapidly during the immediate postnatal period. Indeed, at 2 weeks of age, the craniofacial growth is significantly higher than the growth of the lower limbs. ${ }^{9}$ Generally, the bone growth within a skeletal element can occur at different rates depending on the region considered in the first 6 month after birth, and it is 
influenced by the periosteal, endosteal and osteonic osteogenic fronts. ${ }^{10-12}$ The osteogenic process is a fundamental factor in the understanding of bone growth, and therefore in the evaluation of bone turnover. The efficiency of a biomaterial for bone regeneration is profoundly influenced by the bone remodeling characteristics of the model used to assess it, ${ }^{2}$ therefore an understanding of the underlying mechanisms behind species specific osteogenesis is crucial for the evaluation of new bone regeneration approaches.

The present study aims at evaluating the mineral apposition rate (MAR) of actively growing periosteum, endosteum and osteonic osteogenic fronts within different skeletal elements in New Zealand White rabbits at the age of 6,7 and 8 months. The skeletal elements investigated (femur, tibia, radius, ulna, frontal and parietal bones) were selected considering the anatomical sites commonly used in the regenerative medicine, tissue engineering, and biomaterial field. ${ }^{13-17}$ Our hypothesis is that the MAR varies depending on the skeletal maturity, the skeletal elements, and the osteogenic fronts considered.

Based on evidence from the literature, we hypothesize that the MAR is lower within the osteonic front than in the endosteum and periosteum because of the lower linear density of osteoblast. ${ }^{12}$ We expect a reduction in MAR with aging full skeletal maturity and we hypothesize that MAR varies in the skeletal elements considered due to the different physiological mechanical loading conditions.

\section{Materials and Methods}

\section{Animal procedure}

Nine outbred male New Zealand white rabbits at the same age were randomly selected from three litters and divided into three experimental groups (3x3). The three components of each litter were randomly subdivided in the three experimental groups.

These animals were maintained until the age of 6,7 or 8 months (groups $6 \mathrm{M}, 7 \mathrm{M}$ and $8 \mathrm{M}$, respectively) before sacrifice. At the beginning of the study the animals belonging to groups $6 \mathrm{M}, 7 \mathrm{M}$ and $8 \mathrm{M}$ had mean weights $( \pm \mathrm{SD})$ of $2.95 \pm 0.120,3.030 \pm 0.095$ and $3.39 \pm 0.186 \mathrm{~kg}$, respectively. Animals were housed in separate cages (size 67 X 60 X 35 $\mathrm{cm}$ ), received commercial food (pellets) and water ad libitum and were checked daily. European and Italian regulations on animal experiments were strictly followed during the entire study, according to European Commission regulations. ${ }^{18}$ The experimental procedure and the sample size computation were approved by the Animals Ethics Committee of the University of Parma and by the Health Ministry (REFERENCE). Since the effect due to age, anatomical site and bone structure on osteogenesis investigated in the present research were unknown and no similar reports were available for comparison; thus, the present study can be considered as a pilot experience.

To assess osteogenic activity and MAR, two fluorescent markers were sequentially administered subcutaneously to all animals in the three experimental groups as follows: calcein green (CG; $5 \mathrm{mg} / \mathrm{kg}$ body weight; Sigma, St. Louis, MO, USA), administered daily for 3 days, and xylenol orange (XO; $90 \mathrm{mg} / \mathrm{kg}$ body weight; Sigma), administered daily for 5 days, with a marker interval of 7 days. The colours of these fluorochromes are clearly distinguishable from each other (green and red), and thus they can be used sequentially to highlight newly deposited bone. ${ }^{19}$ Forty-eight hours after the last XO administration, animals were pharmacologically euthanized with Tanax $(0.3 \mathrm{~mL} / \mathrm{kg}$ body weight, Hoechst Roussel Vet, Milan, Italy).

Bone samples were harvested as follows: 1.5 -cm-long sections were collected from the femur distal epiphysis (F1) and the midshaft (F2) (Figure 1a), 1.5-cm-long samples were harvested from the proximal epiphysis (T1) and the midshaft (T2) of the tibia (Figure 1b). As for the radius (R) and ulna (U) 1.5-cm-long samples were harvested from the midshaft of each bone (Figure 1c). We also harvested two samples from the skull of each rabbit: 1.5 -cm wide square tissue samples crossing the sagittal suture, localized cranially respect to the coronal suture for the frontal bone (F) and caudally for the parietal bone (P) (Figure 1e). Together these bone samples form the skeletal elements that were considered for the histomorphometric analysis.

\section{Histomorphometry}

Bone samples were harvested, immediately fixed in $4 \%$ paraformaldehyde, dehydrated in increasing concentration alcoholic solutions (70 up to 100\%), cleared with xylene and finally embedded in polymethylmethacrylate resin (Osteo-Bed; Polysciences, Warrington, PA, USA). Specimens were sequentially cross-sectioned (thickness, 50 $\mathrm{m})$ perpendicularly to the longitudinal axis of the bone using a Leitz 1600 saw microtome (Leitz, Wetzlar, Germany). One section out of four was randomly chosen for subsequent analysis, and three sections for each skeletal elements were used for histomorphometric analysis.

For dynamic histomorphometric measurements, sections were observed under fluorescent microscopy using a motorized microscope (Nikon Eclipse 90i, Nikon, Tokyo, Japan) equipped with a digital camera (Nikon model 5M) and connected to a PC with image analysis software (NIS - Elements AR 3.1, Nikon Tokyo, Japan) comprising a realtime 2D deconvolution module. Mineral apposition rate (MAR) was measured as micrometers per day and calculated from the distance between the corresponding edges of two consecutive labels divided by the time interval between labeling. ${ }^{20}$ Parameter calibrations for magnification and image acquisition were established on the basis of pre-set standardized conditions.

The histomorphometric analysis was performed on the skeletal elements above described. In particular, the MAR of osteonic (0), periosteum (P) and endosteum (E) osteogenic fronts was assessed within $3 \mathrm{~mm}^{2}$ regions of interest (ROIs). Regions of interest in long bones were located in craniolateral (CrL), caudolateral (CaL), craniomedial (CrM) and caudomedial (CaM) position within the cross-sections (Figure 1d). For the skull, ROIs were located in cortical tables (OT), diploe (D) and inner cortical tables (IT) (Figure 1f).

Statistical analysis was performed using IBM SPSS Statistics 19 (IBM Italia S.p.A., Segrate, Milan, Italy). Data were analysed with descriptive statistics followed by repeated measures ANOVA with crossed (experimental times) and nested (skeletal elements and osteogenic fronts) factors. Then, a statistical planned test or post-test with Sidak correction was performed. Collected data are summarized and reported in Table 1 . To verify the power, the statistical power tests have been performed ex post on ANOVA results, by means of both the IBM SPSS Statistics 19 and GPower 3.1.

\section{Results}

For each osteogenic front, two consecutive bone fluorochrome labels (CG and XO) were clearly visible, thus it was possible to quantify the MAR (Figure 2). Analysis of variance showed a highly significant interaction between age and factors associated to bone structure (skeletal elements and osteogenic fronts). The statistical power tests performed ex post on ANOVA for $\leq 0.05$ resulted between 84 and $100 \%$.

\section{Mineral apposition rate based on skeletal elements}

Grouping the data by the skeletal elements (Figure 3a), the MAR was higher in femur (F1, F2), tibia (T1, T2) and parietal bone compared to 
ulna, radius and frontal bone. In the long bones, the MAR was higher in the hindlimb (Membrum pelvinum) samples compared to forelimb (Membrum thoracicum) samples. In particular, in the hindlimb the highest levels of the MAR were observed in F1 compared to F2, T1 and T2, which were extremely homogeneous and such homogeneity was not observed in ulna or radius. In flat bones, parietal and frontal elements were substantially different. The MAR in frontal bone was comparable to that recorded in the samples harvested from the forelimb, whereas the MAR in parietal bone was comparable to the one in the samples from hindlimb. For long bones, the MAR in F1 was significantly higher than in F2 ( $\mathrm{P}=0.002), \mathrm{T} 1(\mathrm{P}=0.004), \mathrm{T} 2(\mathrm{P}=0.001)$, ulna and radius $(\mathrm{P}<0.001)$. Moreover, the MAR in $\mathrm{F} 2, \mathrm{~T} 1$ and $\mathrm{T} 2$ was significantly higher than the MAR in ulna and radius $(\mathrm{P}<0.001)$. The statistics thus confirmed the descriptive analysis, showing a different trend between skeletal elements from forelimb and hindlimb. In the flat bones, a statistically significant difference between frontal and parietal elements was observed $(\mathrm{P}<0.001)$.

\section{Mineral apposition rate based on animal age}

Grouping the data according to animal age (Figure $3 \mathrm{c}$-d) showed that bone growth was not uniform over time. Indeed, the MAR in both F1 and F2 decreased from 6 to 8 months, whereas in both T1 and T2 it reaches the lowest value at 7 months. In contrast to elements of the hindlimb, the MAR of both ulna and radius increased following the aging of the animal, with ulna showing lower MAR compared to radius. For the flat bones, the MAR in the frontal element decreased from 6 to 8 months, whereas in the parietal bone it reached a peak at 7 months. The MAR in F1 was significantly higher both at 6 and 7 months than at
8 months $(\mathrm{P}<0.001)$, whereas in F2 it was significantly higher at 6 months compared to both 7 and 8 months $(\mathrm{P}<0.001)$. In $\mathrm{T} 1$, the decrease in the MAR observed at 7 months was statistically significant $(\mathrm{P}<0.001)$ compared to both 6 and 8 months, whereas in T2 the same decrease at 7 months was not statistically significant. Finally, both ulna and radius showed a positive linear trend from 6 to 8 months, but only the MAR in ulna at 6 month was found to be statistically significant in comparison with both $7(\mathrm{P}=0.002)$ and 8 months $(\mathrm{P}<0.001)$.

\section{Mineral apposition rate by osteogenic fronts}

Evaluating the contribution of distinct osteogenic fronts (osteonic, periosteum and endosteum), the MAR of the osteonic front was constant within the skeletal elements considered and it was the lowest among the fronts investigated (Figure 3b). Overall, the MAR from the osteogenic front showed a different trend between hindlimb and forelimb being higher in F1, F2 and T1 compared to ulna and radius. On the other hand, regardless of the element analysed within the hindlimb, the MAR of the endosteum was higher compared to the periosteum, whereas in the forelimbs it was higher in the periosteum compared to the endosteum. When the influence of the age was considered, it was observed that the MAR in both the endosteum and the periosteum of the hindlimbs decreased from 6 to 8 months, whereas it increased in the forelimbs during the same time span. Moreover, the MAR of the osteogenic fronts within all the skeletal elements considered was not affected by the aging of the animal. In hindlimb, the MAR in the endosteum was significantly different from the MAR in both the periosteum $(\mathrm{P} \leq 0.001)$ and the osteogenic $(\mathrm{P}<0.001)$ front. In the same skeletal elements, a decreasing trend in the contributions of both the endosteum



a)

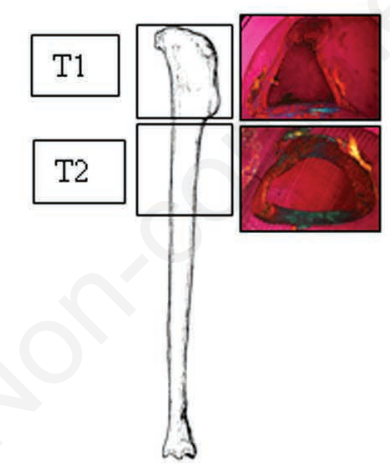

b)

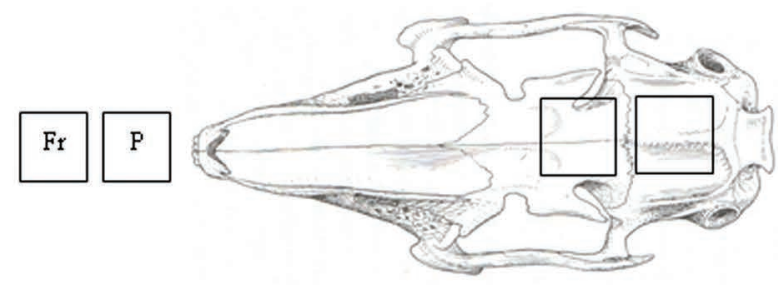

e)

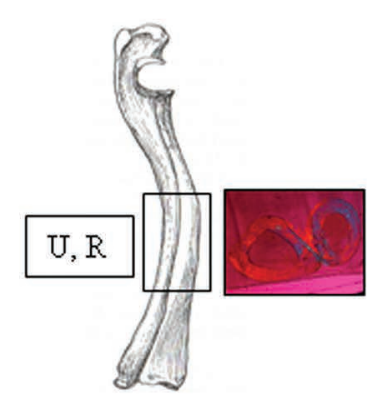

c)

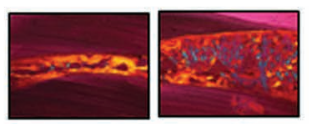



d)

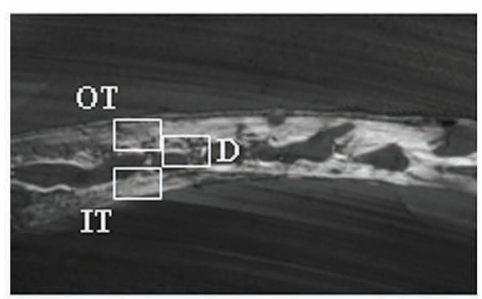

f)

Figure 1. Scheme of the skeletal elements considered in the study and their representative histological cross-sections in linearly polarized light coupled with full-wave compensation: a) femur (F1 and F2); b) tibia (T1 and T2); c) radius (R) and ulna (U); e) frontal (Fr) and parietal $(\mathrm{P})$ bones. Scheme of region of interest considered in the study located in the cross-sections in linearly polarized light, respectively: d) for long bones caudolateral (CaL), caudomedial $(\mathrm{CaM})$, craniolateral $(\mathrm{CrL})$, craniomedial $(\mathrm{CrM})$; f) for flat bones outer cortical tables (OT), diploe (D), and inner cortical tables (IT). Within each region of interest, periosteum, endosteum and osteonic osteogenic fronts were measured and collected. 


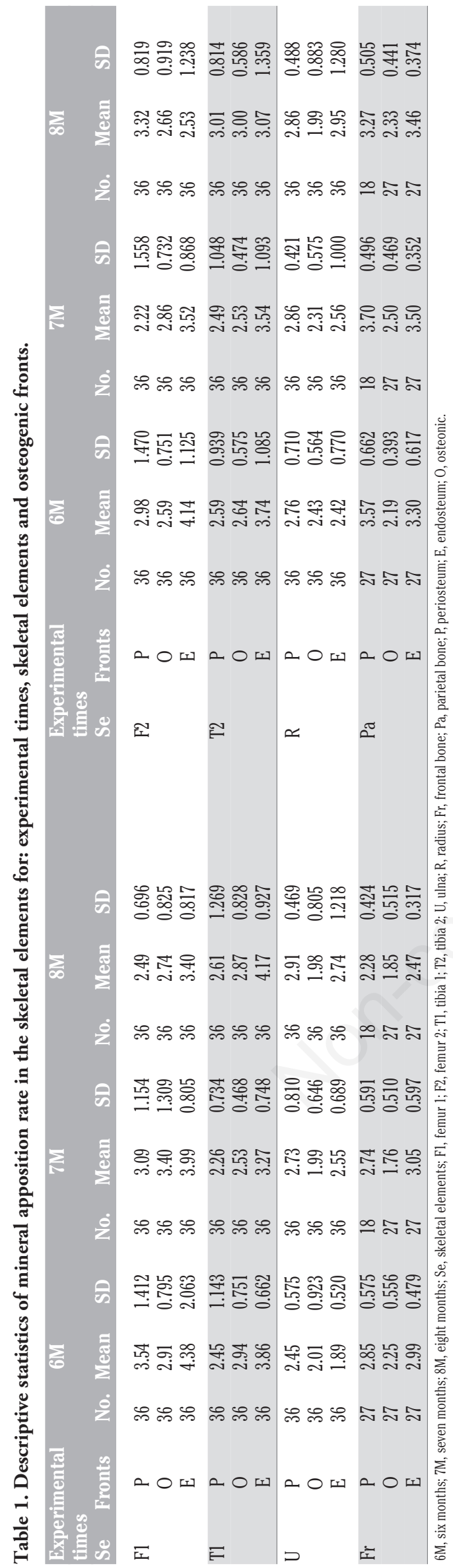

and the periosteum from 6 to 8 months was observed. These trends were statistically significant in $\mathrm{F} 1, \mathrm{~F} 2$ and $\mathrm{T} 2(\mathrm{P}<0.001)$ endosteum and in both $\mathrm{F} 1(\mathrm{P}<0.001)$ and $\mathrm{T} 2(\mathrm{P}=0.020)$ periosteum. In the forelimb, the positive influence of the age was statistically significant in ulna at both the endosteum $(\mathrm{P}<0.001)$ and periosteum $(\mathrm{P}=0.009)$, and in radius at the endosteum $(\mathrm{P}=0.002)$. Finally, in flat bones the MAR in the osteonic front was significantly lower than the one in both the endosteum $(\mathrm{P}<0.001$ in both frontal and parietal bones) and the periosteum $(\mathrm{P}=0.002$ in frontal bone; $\mathrm{P}<0.001$ in parietal bone).

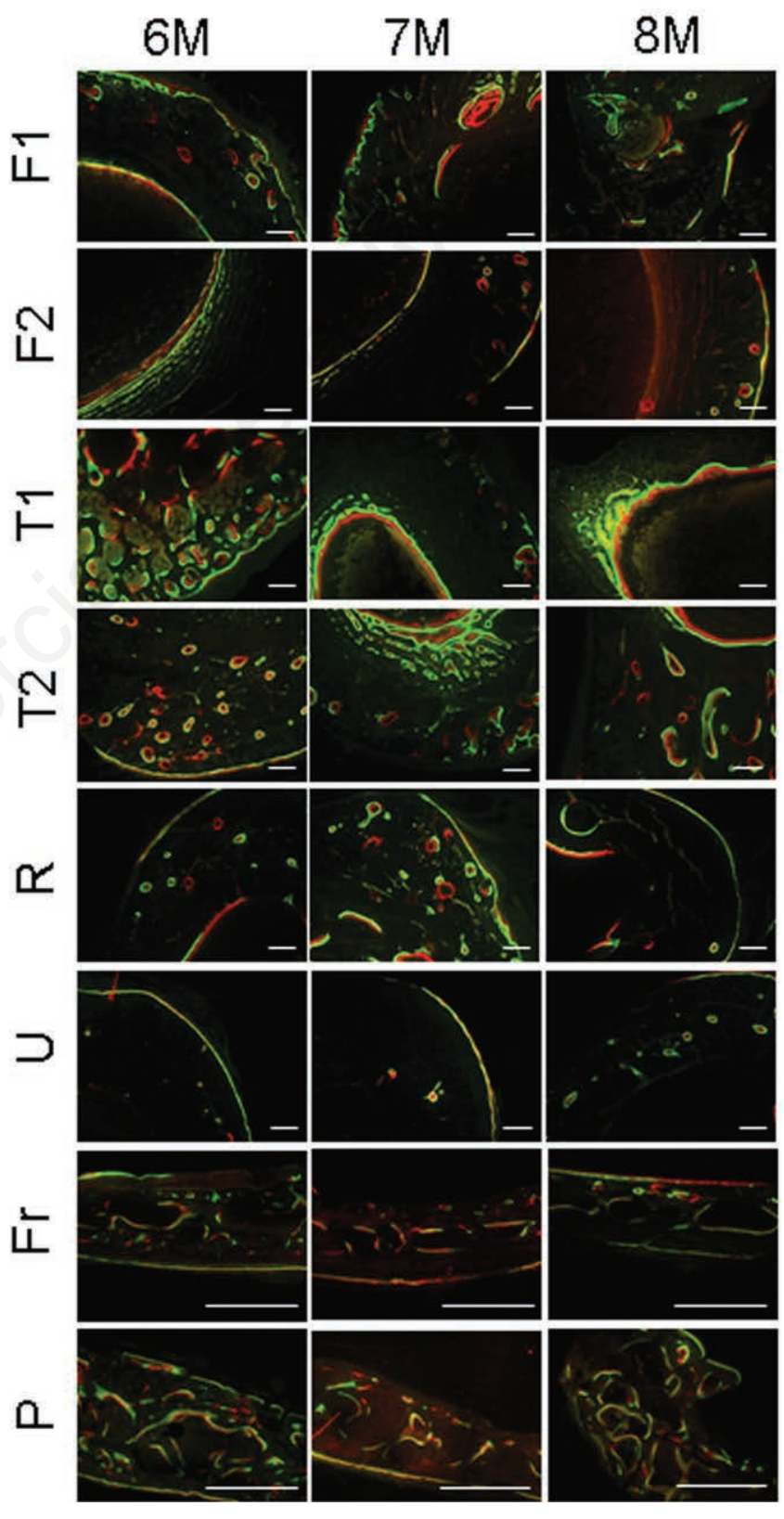

Figure 2. Panel showing calcein green and xylenol orange advancing fronts used for mineral apposition rate assessment. Representative image of each skeletal element (F1, F2, T1, T2, U, $R$, Fr and $P$ ) for all experimental times (6M, $7 M$ and $8 M)$. Scale bars $=250 \mathrm{\mu m}$. F1, distal epiphysis of the femur; F2, corpus of the femur; $T 1$, proximal epiphysis of the tibia; $T 2$, corpus of the tibia; $U$, ulna; $R$, radius; $F r$, frontal; $P$, parietal. 


\section{Discussion}

In most mammals the bone growth is assumed to end briefly after sexual maturity due to growth plate closure. Under physiological conditions, the amount of newly formed and resorbed bone during remodeling is in balance; however, the appositional growth by osteoblasts at the endosteal, the periosteal and the osteonic bone edge occurs at different rates throughout the lifespan.

The objective of the current study was to investigate whether the MARs in New Zealand White rabbits varies according to osteogenic front, skeletal element and age of the animals. Our results demonstrate that the MAR in both femur and tibia is statistically higher than in both ulna and radius, therefore the MAR of skeletal elements from the hindlimb is statistically higher than the MAR of the forelimb elements. It is possible to speculate that these results are due to the higher mechanical load experienced by rabbit hindlimbs. It was shown that the average residual stresses is $210 \mathrm{MPa}$ in hindlimb bones and $140 \mathrm{MPa}$ in forelimb bones respectively, indicating that hindlimb bones are subject to a tensile residual stress 1.4 times higher than forelimb bones. ${ }^{21}$ Relating to the flat bones investigated in the present study, the MAR of the parietal bones is statistically higher compared to the frontal bone, a



C



$\mathrm{b}$

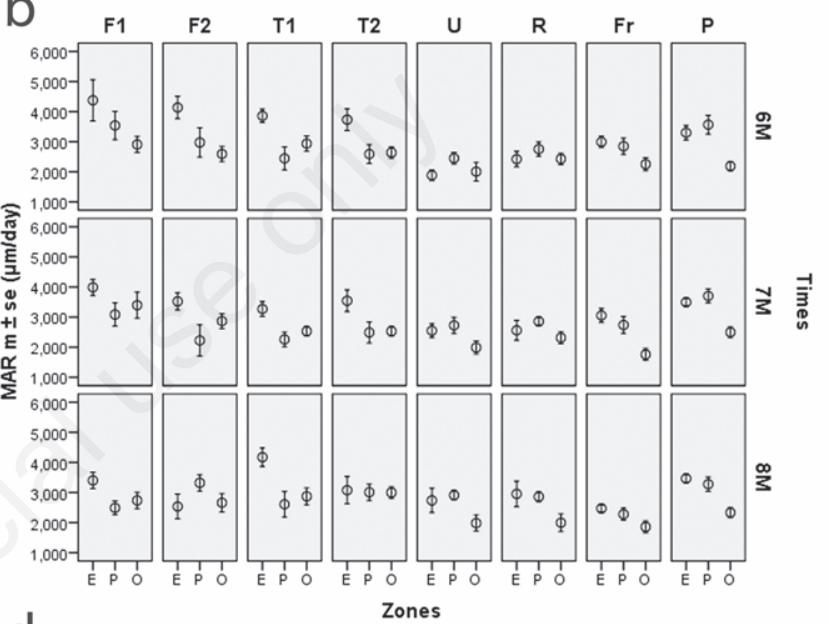

d



Figure 3. a) Mineral apposition rate (MAR) based on skeletal element; b) MAR measured on osteogenic fronts for different skeletal elements at the experimental times of 6,7 , and 8 months $(6 \mathrm{M}, 7 \mathrm{M}$ and $8 \mathrm{M})$, and osteogenic fronts [osteonic $(\mathrm{O})$, periosteum $(\mathrm{P})$ and endosteum (E)]; c-d) MAR based on animal age for different skeletal elements and experimental times 6M, $7 \mathrm{M}$ and $8 \mathrm{M}$ plotted with error graph (c) and line graph (d), respectively. F1, distal epiphysis of the femur; F2, corpus of the femur; T1, proximal epiphysis of the tibia; T2, corpus of the tibia; $U$, ulna; $R$, radius; Fr, frontal; $P$, parietal. 
but it is also analogous to the MAR of both the hindlimb and the forelimb bones. On the other hand, the MAR of the frontal element is similar to the rates observed in the forelimb parts. These results can be explained by the presence of the temporal muscles insertion in the area of the skull investigated. The superficial temporalis muscle is a bipinnate muscle used for mastication, which originates from the dorso-lateral surface of the skull just posterior to the position of the lower jaw articulation, along the border of the parietal and squamosal bones in the fossa for superficial temporalis muscle. It is possible that this muscle influences the mechanical loading of both frontal and parietal bones accounting for the differences found.

Although the association between the mechanical loading and bone (re)modelling was well studied, ${ }^{22,23}$ the mechanisms underlying this phenomenon were just elucidated. Recently, de Jong and colleagues ${ }^{24}$ compared the micro-computed tomography (micro-CT) derived mineraldensity maps of cortical bone from rabbit mandibles to the strain-amplitude maps of the same mandibles predicted by finite-element modelling, reporting a strong correlation between the measured mineral densities and the predicted strains. ${ }^{24}$ Another recent study used time-lapsed in vivo micro-CT to correlate the sites of local bone formation, resorption and quiescence with the local strain distribution calculated by microfinite element (micro-FE) models, showing that local bone formation and resorption are regulated by the application of mechanical loading. ${ }^{25} \mathrm{We}$ speculated a decrease in the MAR following the achievement of full skeletal maturity and therefore the aging of the animal, but surprisingly the results obtained are in contrast with our hypothesis. In both ulna and radius, the MAR increased as the animals aged. On the other hand, for the other skeletal elements considered there is an overall decreasing trend from 6 to 8 months for both the femur elements (F1, F2) and the frontal bone, whereas this trend begins only after 7 months for both the tibia elements (T1,T2) and the parietal bone. Rivas and Shapiro ${ }^{26}$ studied the histological events involved in the formation of long bones in New Zealand White rabbits from the embryonic limb-bud stage to skeletal maturity, identifying various developmental stages and determining the age at which each event occurs. ${ }^{26}$ In particular, the final stages of maturation involve: physis resorption with the linkage of epiphyseal and metaphyseal circulations (stage 16) and the calcification of the lowest zone of articular cartilage, with tidemark formation, and transformation of bone marrow to fat (stage 16a). The authors reported that at 8 months of age both proximal and distal epiphyses of the femur are at stage 16, whereas both proximal and distal epiphyses of the tibia are at stage 16a. Although femur growth plate closes earlier compared to the one in tibia, the skeletal maturation in femur at 8 months of age is at an earlier stage than in tibia. ${ }^{26}$ These results agree and support the different MARs detected within the femur and tibia in this study.

In the present paper we analysed the contributions of different osteogenic fronts to the MAR as both osteoblasts and mesenchymal stem cells. They are recruited for osteoblast differentiation and are present in the periosteum and endosteum. ${ }^{12}$ Our data suggests that for both long and flat bones, the contribution of the osteonic-osteogenic front towards MAR is the lowest and it is not influenced by the different bone architecture that characterises the skeletal element considered. This evidence is supported by the fact that osteoblasts in Haversian canals lay on the surface much longer than in the periosteum, ${ }^{10}$ and also because their linear density is significantly lower in the secondary osteon than in the endosteum. ${ }^{12}$

A previous study aimed at evaluating the MAR in a single cross-section plane within the femur reported differences among the ROIs during the experimental times considered. ${ }^{27}$ Although different ROIs within each skeletal element were selected in this study, we did not focus on the changes in the MAR within these regions as the influences of muscle and tendon insertions were not analysed.

It is known that the natural bone loading generated from the physi- ological functional activity acts as mechanical stimulation ${ }^{28}$ and various studies reported that shear and strain forces modulates proliferation, differentiation, bone remodelling and activation of matrix protein genes in osteoblast cells. ${ }^{28,29}$

\section{Conclusions}

In this study, the influence of both age and osteogenic fronts on bone MAR was demonstrated in different skeletal elements. Skeletal elements often used as sites for bone implants were analysed and the results cast light on the physiological levels of bone turnover in New Zealand White rabbits, providing new insight for the evaluation of bone implants for tissue regeneration. Among the skeletal elements investigated (femur, tibia, radius, ulna, frontal and parietal bones) MAR resulted statistically higher in femur and tibia than in ulna and radius demonstrating that MAR is statistically higher in the skeletal elements from the hindlimb than from the forelimb. Regarding the actively growing periosteum, endosteum and osteonic osteogenic fronts, the contribution of the osteonic front towards the MAR is the lowest. Moreover, the MAR values obtained from each bone analyzed did not decrease uniformly in the different stages examined (6, 7 and 8 months). Both age and implant sites show different MAR values and that should be considered in the development or in the evaluation of a bone graft. The present conclusions could be useful in a dynamic histomorphometry research aimed at investigating bone tissue reparation or regeneration.

\section{References}

1. Neyt JG, Buckwalter JA, Carroll NC. Use of animal models in musculoskeletal research. Iowa Orthop J 1998;18:118-23.

2. Stubinger S, Dard M. The rabbit as experimental model for research in implant dentistry and related tissue regeneration. $\mathrm{J}$ Invest Surg 2013;26:266-82.

3. Gilsanz V, Roe TF, Gibbens DT, et al. Effect of sex steroids on peak bone density of growing rabbits. Am J Physiol 1988;255:e416-21.

4. Van Roermund PM, Haaring C, van Rijk PP, Renooij W. Uptake of [ $99 \mathrm{mTc}$ ]technetium methylene diphosphonate in the growth plates of the rabbit tibia during the final part of epiphyseal growth activity. Acta Orthop Belg 1994;60:369-73.

5. Kilborn SH, Trudel G, Uhthoff H. Review of growth plate closure compared with age at sexual maturity and lifespan in laboratory animals. Contemp Top Lab Anim 2002;41:21-6.

6. Kaweblum M, Aguilar MC, Blancas E, et al. Histological and radiographic determination of the age of physeal closure of the distal femur, proximal tibia, and proximal fibula of the New Zealand white rabbit. J Orthop Res 1994;12:747-9.

7. Lowrance E. Roentgenographic record of growth of the femur of the rabbit. Growth 1955;19:247-55.

8. Khermosh 0, Tadmor A, Weissman SL, et al. Growth of the femur in the rabbit. Am J Vet Res 1972;33:1079-82.

9. Masoud I, Shapiro F, Moses A. Longitudinal roentgencephalometric study of the growth of the New Zealand white rabbit: cumulative and biweekly incremental growth rates for skull and mandible. $\mathrm{J}$ Cran Genet Dev Bio 1986;6:259-87.

10. Owen M. Cell population kinetics of an osteogenic tissue. I. J Cell Biol 1963;19:19-32.

11. Owen M, Macpherson S. Cell population kinetics of an osteogenic tissue. II. J Cell Biol 1963;19:33-44. 
12. Pazzaglia UE, Congiu T, Franzetti E, et al. A model of osteoblastosteocyte kinetics in the development of secondary osteons in rabbits. J Anat 2012;220:372-83.

13. Yoon SJ, Park KS, Kim MS, et al. Repair of diaphyseal bone defects with calcitriol-loaded PLGA scaffolds and marrow stromal cells. Tissue Eng 2007;13:1125-33.

14. Lee LT, Kwan PC, Chen YF, Wong YK. Comparison of the effectiveness of autologous fibrin glue and macroporous biphasic calcium phosphate as carriers in the osteogenesis process with or without mesenchymal stem cells. J Chin Med Assoc 2008;71:66-73.

15. Cacchioli A, Ravanetti F, Bagno A, et al. Human vitronectin-derived peptide covalently grafted onto titanium surface improves osteogenic activity: a pilot in vivo study on rabbits. Tissue Eng Pt A 2009;15:2917-26.

16. Ravanetti F, Borghetti P, De Angelis E, et al. In vitro cellular response and in vivo primary osteointegration of electrochemically modified titanium. Acta Biomater 2010;6:1014-24.

17. Khojasteh A, Behnia H, Dashti SG, Stevens M. Current trends in mesenchymal stem cell application in bone augmentation: a review of the literature. J Oral Maxil Surg 2012;70:972-82.

18. European Commission, 2010. Directive of the European Parliament and of the Council of 22 September 2010 on the protection of animals used for scientific purposes, 2010/63/EU. In: Official Journal, L 276/33, 20/10/2010.

19. van Gaalen SM, Kruyt MC, Geuze RE, et al. Use of fluorochrome labels in in vivo bone tissue engineering research. Tissue Eng Pt B Rev 2010;16:209-17.

20. Dempster DW, Compston JE, Drezner MK, et al. Standardized nomenclature, symbols, and units for bone histomorphometry: a
2012 update of the report of the asbmr Histomorphometry Nomenclature Committee. J Bone Miner Res 2013;28:1-16.

21. Yamada S, Tadano S, Fujisaki K. Residual stress distribution in rabbit limb bones. J Biomech 2011;44:1285-90.

22. Rubin C, Gross T, Qin YX, et al. Differentiation of the bone-tissue remodeling response to axial and torsional loading in the turkey ulna. J Bone Joint Surg Am 1996;78:1523-33.

23. Skedros JG, Dayton MR, Sybrowsky CL, et al. Are uniform regional safety factors an objective of adaptive modeling/remodeling in cortical bone? J Exp Biol 2003;206:2431-9.

24. de Jong WC, van Ruijven LJ, Brugman P, Langenbach GE. Variation of the mineral density in cortical bone may serve to keep strain amplitudes within a physiological range. Bone 2013;55:391-9.

25. Schulte FA, Ruffoni D, Lambers FM, et al. Local mechanical stimuli regulate bone formation and resorption in mice at the tissue level. PLoS One 2013;8:e62172.

26. Rivas R, Shapiro F. Structural stages in the development of the long bones and epiphyses: a study in the New Zealand white rabbit. J Bone Joint Surg Am 2002;84:85-100.

27. Cacchioli A, Ravanetti F, Soliani L, Borghetti P. Preliminary study on the mineral apposition rate in distal femoral epiphysis of New Zealand white rabbit at skeletal maturity. Anat Histol Embryol 2012;41:163-9.

28. Rubin J, Murphy TC, Rahnert J, et al. Mechanical inhibition of RANKL expression is regulated by H-Ras-GTPase. J Biol Chem 2006;281:1412-8.

29. Rubin CT, Lanyon LE. Limb mechanics as a function of speed and gait: a study of functional strains in the radius and tibia of horse and dog. J Exp Biol 1982;101:187-211. 